\title{
Outcomes of urinary tract abnormalities diagnosed by the routine third trimester scan
}

Spyros Bakalis MBBS BSc MD MRCOG ${ }^{1 *}$

Kevin Cao MBBS MRCS BSc ${ }^{2}$

Radha Graham MBBS, MROCG 3

Peter Cuckow MBBS PhD FRCS FRCS (Paed.) ${ }^{2}$

Nav Johal MB BCh MRCS PhD FRCS (Paed.) ${ }^{2}$

Paul Winyard BA MB BCh MRCP 4

Pranav Pandya MBBS BSc MD FRCOG ${ }^{5}$

Divyesh Desai MBBS FRCS (Urol) ${ }^{3}$

1. Department of Obstetrics, Fetal and Maternal Medicine, Guy's and St Thomas NHS Trust, London

2. Department of Urology, Great Ormond Street Hospital for Children, London

3. Department of Obstetrics and Gynaecology, Homerton University Hospital, London

4. Department of Nephrology, Great Ormond Street Hospital for Children, London

5. Fetal Medicine Unit, University College London Hospital, London

${ }^{*}$ Corresponding author, FMU, $8^{\text {th }}$ floor North Wing, St Thomas' Hospital, Westminster Bridge Road, London, SE1 7EH, UK. spyros@bakalis.net 


\section{Abstract}

Objective: The aim of this study is to determine the incidence of congenital abnormalities of the kidneys and urinary tract (CAKUT) detected for the first time in an unselected population undergoing a routine third trimester scan between 30-34 week's gestation.

Methods: This was a retrospective analysis of 8562 routine third trimester ultrasound scans during which the fetal anatomy was evaluated, and, any structural abnormalities detected, recorded onto a fetal database which was subsequently analysed for CAKUT. All postnatal records of antenatally diagnosed CAKUT were obtained and analysed for diagnosis and management.

Results: There were 26 cases of urological abnormalities detected for the first time in the third trimester. The most frequent abnormality was unilateral renal pelvis dilatation (73\%). Postnatal ultrasound confirmed abnormalities in 19 (73\%) newborns, with two (8\%) resolving antenatally and four (15\%) postnatally. The overall incidence of new CAKUT detected by the third trimester scan was $0.22 \%(19 / 8562)$ with a male to female ratio of $1: 1.6$. Four patients required surgery, two received cystoscopic injection of Deflux with circumcision, one received cystoscopic valve ablation and one patient received a staged hypospadias repair.

Conclusion: Routine third trimester scanning is already performed in many countries with proposed benefits primarily directed towards the monitoring of fetal growth and late pregnancy malpresentation. For healthcare systems that still utili se two routine scans, debate is ongoing as to the value of introducing a routine third trimester scan. The ability to detect additional and potentially missed CAKUT is a further benefit, which in isolation is likely of too small an impact to merit implementation. However, the combination of fetal structural assessment, growth velocity monitoring and fetal presentation evaluation presents a strong case for inclusion in an antenatal screening program. The findings of this study highlight the importance of a detailed fetal structural evaluation at each antenatal ultrasound scan. 


\section{Introduction}

Congenital abnormalities of the kidneys and urinary tract (CAKUT) are quoted as constituting $20-25 \%$ of all fetal structural abnormalities (1) and are detected by prenatal ultrasound in 0.1 $0.4 \%(2)$ of all fetuses. Antenatal detection improves renal outcomes preventing further kidney damage. Recent evidence suggests that $60 \%$ of infants having surgery for CAKUT are detected antenatally (3).

In the UK, the vast majority of these abnormalities are detected during the routine second trimester scan at 18+0-20+6 weeks gestation. Third trimester scanning has been used as a tool to detect fetal growth disorders. There is some literature assessing the incidence and outcome of CAKUT diagnosed in the third trimester, although, this evidence is not from routine uncomplicated pregnancies $(4,5)$.

The aim of this study is to determine the incidence of CAKUT detected for the first time in an unselected population undergoing a routine third trimester scan.

\section{Patients and methods}

A routine third trimester scan at $30+0$ to $34+6$ weeks of gestation was introduced at University College London Hospital (UCLH). The primary aim of the scan was the detection of the small for gestational age fetus. At the time of the scan, fetal biometry, amniotic fluid volume, and Doppler studies of the middle cerebral and umbilical arteries were measured. In addition to fetal biometry the fetal brain, heart, gastrointestinal and urinary systems were routinely revaluated in all cases. Data were consistently entered onto a computerised database which also archived images. The computerised database was searched to identify all new CAKUT detected for the first time in the third trimester between the $1^{\text {st }}$ April 2012 and the $1^{\text {st }}$ February 2014. The second trimester scan for each case was reviewed to ensure that the findings were new to the third trimester. The records of postnatal management at the Great Ormond Street Hospital were examined to determine clinical outcomes.

\section{Antenatal detection}


Antenatal scanning at UCLH is undertaken by experienced fetal sonographers, who refer new fetal structural abnormalities for review in the Fetal Medicine Unit (FMU).

An APD of $10-15 \mathrm{~mm}$ was considered mild, $15-20 \mathrm{~mm}$ moderate and more than $20 \mathrm{~mm}$ severe. The presence, filling and wall thickness of the fetal bladder, presence, morphology (horseshoe, duplex, etc.) and measurement of the longitudinal, transverse and anteroposterior dimensions of each kidney and the antero-posterior diameter (APD) of the renal pelvis were recorded.

All CAKUT cases were counselled jointly by a Fetal Medicine specialist and a paediatric renal physician or urologist. Antenatal follow-up was monthly post diagnosis, except for mild ANH which was discharged from antenatal care. All new cases of fetal abnormalities were routinely referred from the FMU to the neonatal unit for immediate postnatal reassessment and management, whilst long term follow up was conducted by the Paediatric Urologists at Great Ormond Street Hospital.

\section{Postnatal treatment}

Postnatally, neonates with antenatal CAKUT are managed initially at UCLH where they are scanned after five days (or immediately if suspicion of acute obstructive uropathy). Those patients with APD of $<10 \mathrm{~mm}$ were discharged from further urology follow-up. Patients with confirmed unilateral hydronephrosis over $10 \mathrm{~mm}$ are started on prophylactic antibiotics for possible vesico-ureteric reflux and booked for further renal sonography at 6 weeks to 6 months at GOSH. Patients with hydroureteronephrsis were evaluated with further scans, including nuclear medicine scintigraphy and micturating cystourethrogram (MCUG) to look for obstructive pathology. Finally, patients with bilateral hydronephrosis are worked up for acute investigation and management of bladder outlet obstruction (BOO), which usually entails an acute transfer to GOSH to receive cystoscopy. Management pathway for various antenatal CAKUT cases can be found in figures 1. Decision to list patients for further observation, antibiotic treatment or surgery was based on the results of subsequent investigations. 


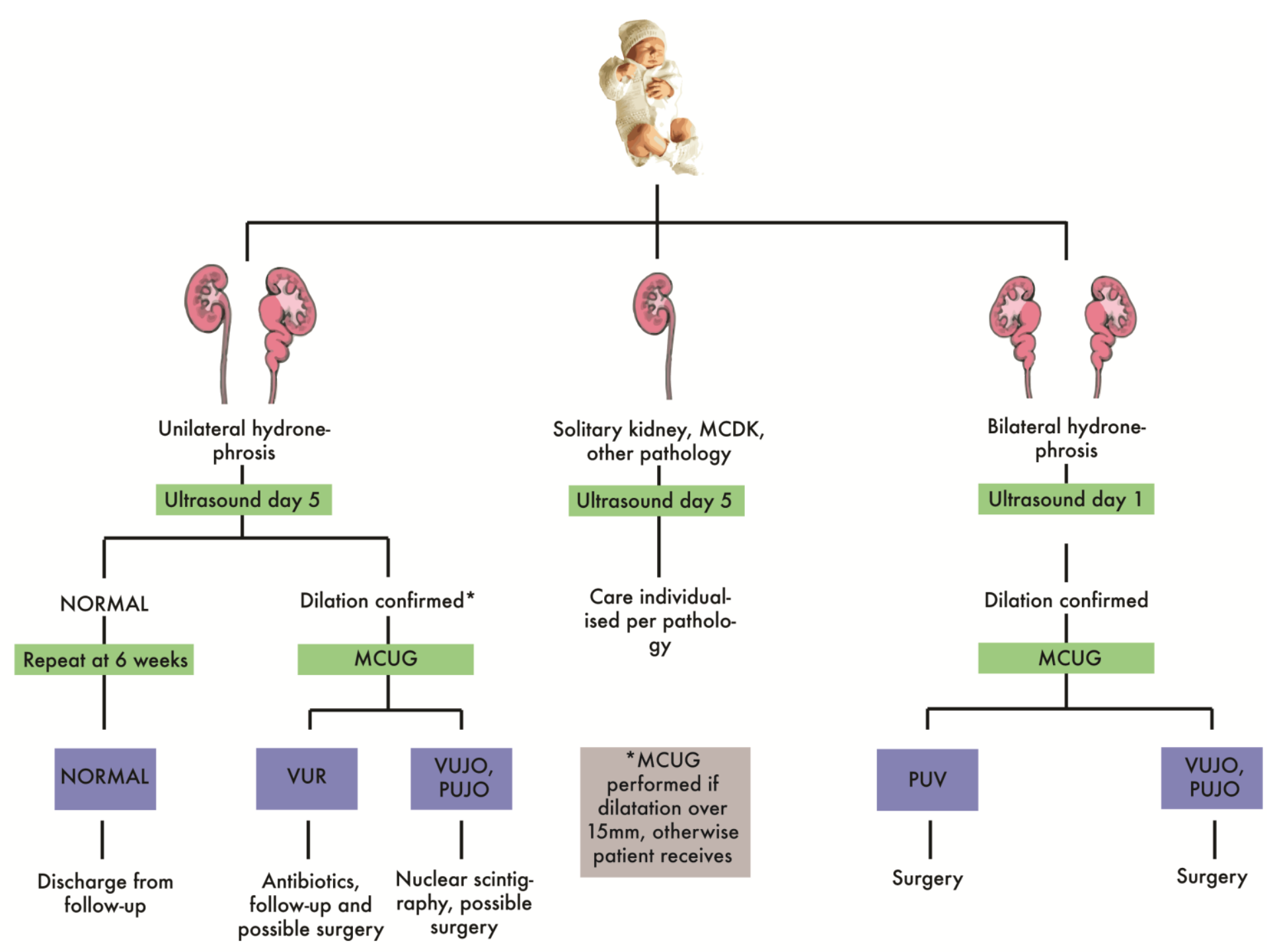

Figure 1. Template for postnatal management of antenatally detected urologic abnormality. Micturating cystourethrogram (MCUG), vesico-ureteric reflux (VUR), vesico-ureteric junction obstruction (VUJO), pelvi-ureteric junction obstruction (PUJO), multicystic dysplastic kidney (MCDK), and posterior urethral valves (PUV). 


\section{Results}

Third trimester scan

There was a total of 8562 routine third trimester scans carried out in singleton pregnancies with no known fetal abnormalities. The mean maternal age was 32.7 and 31.0 years in the normal and CAKUT groups respectively. The gestational age of the scan was between $30+0$ to $34+6$ weeks, with a mean of gestational age of 32.9 weeks in the normal and 33.0 in the CAKUT groups. The maternal characteristics are shown in table 1.

Table 1. Maternal characteristics in the normal and CAKUT groups. Data are given as median (interquartile range) or $n(\%)$.

\begin{tabular}{|l|l|l|}
\hline Characteristic & Normal $(\mathrm{n}=8536)$ & CAKUT $(\mathrm{n}=26)$ \\
\hline Maternal age & $32.7(29.6-36.4)$ & $31.0(32.4-33.7)$ \\
\hline Maternal weight & $73.0(66.5-82.0)$ & $69.7(65.3-76.4)$ \\
\hline Maternal height & $164.2(160.0-169.0)$ & $166.0(162.0-170.0)$ \\
\hline GA at screening & $32.9(32.1-33.7)$ & $33.0(32.4-33.7)$ \\
\hline Racial origin & & \\
\hline$\bullet \quad$ Caucasian & $6134(71.9)$ & $22(84.6)$ \\
\hline$\bullet$ Afro-Caribbean & $866(10.1)$ & $2(7.7)$ \\
\hline$\bullet$ South Asian & $815(9.5)$ & $1(3.8)$ \\
\hline$\bullet$ East Asian & $493(5.8)$ & $0(0)$ \\
\hline$\bullet$ Mixed & $228(2.7)$ & $1(3.8)$ \\
\hline$\bullet$ Smoking & $191(2.3)$ & $0(0)$ \\
\hline
\end{tabular}

\section{Antenatal diagnosis}

There were 26 cases of urological abnormalities detected for the first time in the third trimester, which are listed in table 1 . The most frequent abnormality was unilateral renal pelvis dilatation (73\%) followed by bilateral renal pelvis dilatation (12\%) duplex renal system (8\%), unilateral renal agenesis (4\%) and renal cyst (4\%) (see table 2). 
Table 2. Genitourinary abnormalities detected by the third trimester scan

\begin{tabular}{|c|c|}
\hline Genitourinary Abnormality & $\mathbf{n}(\%)$ \\
\hline Unilateral pelvis dilatation & $16(73 \%)$ \\
\hline Bilateral pelvis dilatation & $3(12 \%)$ \\
\hline Absent kidney & $1(4 \%)$ \\
\hline Duplex & $2(8 \%)$ \\
\hline Other & $4(15 \%)$ \\
\hline TOTAL & $26(100 \%)$ \\
\hline
\end{tabular}

Postnatal diagnosis confirmation

Postnatal ultrasound confirmed abnormalities in 19 (73\%) newborns, with 2 (8\%) resolving antenatally and 4 (15\%) resolving on their first postnatal scan. The overall incidence of new genito-urinary abnormalities detected by the third trimester scan was $0.22 \%(19 / 8562)$ with a positive predictive value of $76 \%$ (see table 2). The male to female ratio in our study was $1: 1.6$, i.e. a $50 \%$ greater number of females with proven urologic pathology. The most common diagnosis was physiologic antenatal hydronephrosis (ANH) $(31 \%)$ followed by VUR (12\%), unilateral renal agenesis (8\%), posterior urethal valves (4\%), megaureter (8\%) and penile hypospadias (4\%). One neonate was lost-to-follow-up (4\%). The full list of postnatal diagnoses is listed in Table 3.

Table 3. Postnatal diagnoses from newly-detected abnormalities at the third trimester scan

\begin{tabular}{|l|l|}
\hline Postnatal diagnoses & $\mathbf{n}(\%)$ \\
\hline Resolved & $6(23)$ \\
\hline$-\quad$ Antenatally & $2(8)$ \\
\hline$-\quad$ Postnatally & $4(15)$ \\
\hline Agenesis & $2(8)$ \\
\hline PUJ & 0 \\
\hline Duplex & $2(4)$ \\
\hline Valves & $1(4)$ \\
\hline
\end{tabular}




\begin{tabular}{|c|l|}
\hline Megaureter & $2(8)$ \\
\hline VUR & $3(12)$ \\
\hline$-\quad$ Bilateral & $2(8)$ \\
\hline$-\quad$ Unilateral & $1(4)$ \\
\hline Hydronephrosis & $8(31)$ \\
\hline$-\quad$ Bilateral & $1(4)$ \\
\hline$-\quad$ Unilateral & $7(27)$ \\
\hline Other & $1(4)$ \\
\hline & \\
\hline Lost to follow up & $1(4)$ \\
\hline & \\
\hline Incidence & $0.22 \%$ \\
\hline
\end{tabular}

\section{False negatives}

There were an additional seven cases of ANH which were identified as 'new' urologic abnormalities by the routine third trimester scan. However, on retrospective evaluation of second trimester scans, these cases were identified as normal, despite ANH already being present. These cases were therefore incorrectly identified as normal in the second trimester. If these patients were included as newly detected third trimester cases, then the total number of abnormalities would increase to 33 or an incidence of $0.33 \%$.

\section{Postnatal management}

\section{Vesico-ureteric reflux (VUR)}

The three patients with VUR received antibiotic prophylaxis, with two cases requiring antireflux surgery (Deflux@ injection). Follow-up was discontinued based on the results of sequential scans.

\section{Urinary tract infection}

Urinary tract infection is one of the principle presentations of neonatal urological disease along with obstruction and abdominal masses. In our group only one patient had infection, 
having an underlying postnatal diagnosis of non-refluxing megaureter.

\section{Other renal anomalies}

The two cases of megaureter received antibiotic prophylaxis for six months whilst the patients with unilateral agenesis were followed up for 6 months without treatment.

\section{Other urologic anomalies}

One patient had posterior urethral valves and one proximal hypospadias. The former received a valve ablation procedure and the latter a staged repair.

\section{Surgery}

Four patients required surgery. Two patients received cystoscopic injection of Deflux with circumcision, one received cystoscopic valve ablation and one patient received a staged hypospadias repair.

Though these are technically not newly diagnosed third trimester abnormalities, in the absence of a routine third trimester scan they would not have been identified antenatally. Of the seven cases (see table 4) 3 patients requires surgery ( 1 pyeloplasty and 2 circumcisions) and 1 had a UTI.

Table 4. Postnatal outcomes of ANH misidentified as normal at the second trimester

\begin{tabular}{|l|l|}
\hline Pathology & n \\
\hline Vesico-ureteric reflux & 1 \\
\hline Megaureter & 1 \\
\hline Hydronephrosis & 2 \\
\hline PUJ stenosis & 2 \\
\hline Lost to follow up & 1 \\
\hline
\end{tabular}




\section{Discussion}

The main finding of our study is that routine third trimester scanning is able to detect additional urologic abnormalities not present at the second trimester scan, whose identification can lead to early postnatal intervention and hence reduction in long term co-morbidities. Our study is unique three ways. Firstly, it looks at all possible CAKUT in the third trimester only, unlike similar studies which includes second and third trimester findings. Secondly, it uses a larger APD cut off of $10 \mathrm{~mm}$ rather than $7 \mathrm{~mm} \mathrm{(5)}$ therefore allowing us to decrease the false positive rate. Thirdly, ours is a true routine population and does not rely on referrals a from other centres, which would increase the incidence of pathology.

Our data suggests that the third trimester has a higher detection rate of true postnatal urologic pathology (73\%) and a lower rate of spontaneous resolution $(26 \%)$ compared to second trimester ultrasound, where resolution rates of up to $50 \%$ are seen in the literature, especially when APD is below $10 \mathrm{~mm}(6)$. The additional ultrasound findings increase the postnatal CAKUT rate by $0.3 \%$. A study by Shipp et al. regarding routine third trimester scanning found a postnatal CAKUT incidence of $1.8 \%$ (5). The lower incidence in our cohort is mostly explained by the difference in APD thresholds used (7mm in Shipp et al. (5) and 10mm by us) as well as how false negative cases were handled. Fetuses with apparent new anomalies at the third trimester scan but on review found to be abnormal at the second trimester scan were included as new cases by Shipp. In our analysis, we excluded these although in practice, these fetuses form part of the false negative rate of the third trimester scan and would have increased our CAKUT incidence by $0.4 \%$. Other studies on third trimester scanning have quoted higher rates of CAKUT diagnoses, however these are in unselected populations $(4,5)$.

A frequent finding in observational research is that ANH is more prevalent in males (up to 4:1 ratio) and more often left-sided (2:1) (7-10). Studies on third trimester scanning in selected populations uphold these observational patterns. In our cohort, there was a minor female and right-sided ANH predominance. A similar slight female predominance was also found by Ek et al., (1:1.5 male to female ratio), who investigated third trimester scanning in a routine population (11). We speculate that the apparent male predominance seen with studies looking at second trimester scanning, may be a phenomenon seen only earlier in gestation that does not translate to postnatal pathology. Perhaps the greater outflow tract resistance in 
males can explain this pattern in earlier gestation and its disappearance may be explained by the increase in the capacity of the fetal bladder.

The precise threshold for detecting ANH determines the sensitivity and specificity at the second and third trimester scan. Two systems exist for this categorisation, the Society of Fetal Urology (SFU) grade and the Antero-posterior diameter (APD), with most sonographers and literature reporting in the latter format. There is debate as to what level to set as a threshold for detection, with some studies suggesting that $7 \mathrm{~mm}$ at the third trimester $(6,12,13)$, whilst others opt for $10 \mathrm{~mm}$. We used a threshold of $10 \mathrm{~mm}$ at the third trimester scan, as this is more closely aligns with that used after birth. We felt this value provided a superior compromise between excess detection of benign ANH without missing real pathology.

An important question surrounding routine third trimester scanning is the value of the detected pathology, or whether there is any benefit in making these additional detections. Unfortunately, this research cannot conclusively settle this debate. There are many proponents of a more conservative approach toward antenatal urologic abnormalities with research suggesting that lower grades of APD $(5-7 \mathrm{~mm}$ at second trimester, $<10 \mathrm{~mm}$ at third trimester) often do not translate to real pathology. This 'benign' hydronephrosis is a group that is arguably over investigated and over-treated. Many urologists prescribe prophylactic antibiotics for these patients, but others advocate a more cautious approach with prophylaxis as urinary tract infections (UTI) are relatively uncommon in patients with mild hydronephrosis $(7,14)$. There is no consensus regarding the correct management of these patients, with some advocating the use of multiple predictive factors such as higher grade hydronephrosis, bilateral hydronephrosis and renal scarring to determine whom to give antibiotics to $(7,15)$. However, what is clear is that patients with higher grades of APD (quoted as over $10 \mathrm{~mm}$ or $15 \mathrm{~mm})$ are more likely to need surgery $(6,13,16)$. The literature also supports the finding that ANH discovered in the third trimester has a higher rate of UTI than those detected earlier (14), potentially altering the risk profile of these children.

A more convincing argument against third trimester scanning is that findings such as megaureter and solitary kidneys are not clinically concerning. The literature suggests that primary megaureter is the cause of $6-10 \%$ of all ANH (17), but there is a higher than normal rate of surgery in this group, with one study finding that $22 \%$ of these patients ultimately had 
reimplantation of their ureters (8). Whilst there is little that can be done for patients with a solitary kidney, it is recognised that there is a high co-abnormality rate and an association between solitary kidney and early-onset hypertension (14). Arguably, the pathologies found by the routine third trimester scan are not inconsequential, with seven patients in our group requiring surgery, three of which were newly discovered whilst four were misidentified as normal at the second trimester study. Routine third trimester scanning thus detects latepresenting pathology and is a second chance to pick up missed pathology from earlier scans.

The biggest argument against employing a routine third trimester scan purely to detect additional fetal abnormalities is one of Pareto efficiency, or rather the optimal use of resources in a healthcare system. It would be very hard, if not impossible to make a case that detecting additional CAKUT should divest resources from other more impactful healthcare screening policies, especially when third trimester CAKUT will generally not change the course of pregnancy management and may carry with it the potential for unwarranted anxiety and possibly even overtreatment. This may seem to rule out the value of third trimester scanning for fetal structural pathology particularly as the absolute diagnosis rate of CAKUT is so low. But we must remember that the CAKUT diagnosis rate from the established-second trimester scan is also very low and the value of obstetric scanning lies firstly with its utility in monitoring fetal growth, presentation and screening for maternal pregnancy-related disorders. In these areas, a growing body of research is demonstrating that the third trimester scan may be of significant benefit, in picking up many more small-for-gestational age fetuses and detecting pre-eclampsa $(18,19)$. In this regard, having the additional window of opportunity to screen for CAKUT, provide counselling and preparation for postnatal investigation and in some cases pre-empting of kidney damage becomes a beneficial corollary of scanning in the third trimester.

The limitations of this study were threefold. Firstly, it was a retrospective analysis of the antenatal and postnatal clinical outcomes of a cohort of patients scanned with a routine third trimester scan at UCLH. It was not a randomised controlled trial; thus, all patients were exposed to this screening intervention and there is no technical control group. We have therefore made our comparisons against literature from other centres. Secondly that we only reviewed the clinical notes of all antenatally detected CAKUT patients for up to 3 years. Thirdly, we were not able to scan normal patients after delivery in order to evaluate the full sensitivity and specificity of routine third trimester scanning in our population. However, as discussed previously, this would have been a sizable endeavour to undertake with this 
population size. We did consider sampling our 'normal' population, however, as the incidence of CAKUT that only presents postnatally is an unknown entity, the necessary sample size for review would beyond the capacity of the children's hospital to offer.

\section{$\underline{\text { Conclusion }}$}

Routine third trimester scanning is already performed in many countries with benefits primarily directed towards the monitoring of fetal growth and late pregnancy malpresentation. For healthcare systems that still utilise two routine scans, debate is ongoing as to the value of introducing an additional scan. The additional ability to detect potentially missed CAKUT is an added benefit, which in isolation is likely of too small an impact to merit implementation of a third trimester scan, but in concert with other proposed benefits such as monitoring for late fetal growth velocity, malpresentation and screening for maternal disease, makes a strong case for implementing routine third trimester scanning. In addition, it highlights the need for a detailed fetal structural evaluation at all scans. 


\section{References}

1. Elder JS. Antenatal hydronephrosis. Fetal and neonatal management. Pediatr Clin North Am. 1997 Oct;44(5):1299-321.

2. Grandjean $\mathrm{H}$, Larroque $\mathrm{D}$, Levi $\mathrm{S}$. The performance of routine ultrasonographic screening of pregnancies in the Eurofetus Study. Am J Obstet Gynecol. 1999 Aug;181(2):446-54.

3. Bhide A, Sairam S, Farrugia M-K, Boddy S-A, Thilaganathan B. The sensitivity of antenatal ultrasound for predicting renal tract surgery in early childhood. Ultrasound Obstet Gynecol. 2005 May;25(5):489-92.

4. Wollenberg A, Neuhaus TJ, Willi UV, Wisser J. Outcome of fetal renal pelvic dilatation diagnosed during the third trimester. Ultrasound Obstet Gynecol. 2005 May;25(5):483-8.

5. Shipp TD, Nguyen HT, Bromley B, Lyons JG, Benacerraf BR. Importance of renal abnormalities first identified in the third trimester after normal findings on a detailed second-trimester structural fetal survey. J Ultrasound Med. 2011 Nov;30(11):156772.

6. Hothi DK, Wade AS, Gilbert R, Winyard PJD. Mild fetal renal pelvis dilatation: much ado about nothing? Clin J Am Soc Nephrol. 2009 Jan;4(1):168-77.

7. Alconcher LF, Tombesi MM. Natural history of bilateral mild isolated antenatal hydronephrosis conservatively managed. Pediatr Nephrol. 2012 Jul;27(7):1119-23.

8. Arena S, Magno C, Montalto AS, Russo T, Mami C, Baldari S, et al. Long-term followup of neonatally diagnosed primary megaureter: rate and predictors of spontaneous resolution. Scand J Urol Nephrol. 2012 Jun;46(3):201-7.

9. Chertin B, Pollack A, Koulikov D, Rabinowitz R, Hain D, Hadas-Halpren I, et al. Conservative treatment of ureteropelvic junction obstruction in children with antenatal diagnosis of hydronephrosis: lessons learned after 16 years of follow-up. Eur Urol. $2006 \mathrm{Apr} ; 49(4): 734-8$.

10. Kim HJ, Jung HJ, Lee HY, Lee YS, Im YJ, Hong CH, et al. Diagnostic value of anteroposterior diameter of fetal renal pelvis during second and third trimesters in predicting postnatal surgery among Korean population: useful information for antenatal counseling. Urology. 2012 May;79(5):1132-7.

11. Ek S, Lidefeldt K-J, Varricio L. Fetal hydronephrosis; prevalence, natural history and postnatal consequences in an unselected population. Acta Obstet Gynecol Scand. 2007;86(12):1463-6.

12. Grazioli S, Parvex P, Merlini L, Combescure C, Girardin E. Antenatal and postnatal ultrasound in the evaluation of the risk of vesicoureteral reflux. Pediatr Nephrol. 2010 Sep;25(9):1687-92. 
13. Policiano C, Djokovic D, Carvalho R, Monteiro C, Melo MA, Graca LM. Ultrasound antenatal detection of urinary tract anomalies in the last decade: outcome and prognosis. J Matern Fetal Neonatal Med. 2015 May;28(8):959-63.

14. Becker AM. Postnatal evaluation of infants with an abnormal antenatal renal sonogram. Curr Opin Pediatr. 2009 Apr;21(2):207-13.

15. Walsh TJ, Hsieh S, Grady R, Mueller BA. Antenatal hydronephrosis and the risk of pyelonephritis hospitalization during the first year of life. Urology. 2007 May;69(5):970-4.

16. Kiener TA, Wohlmuth C, Schimke C, Brandtner MG, Wertaschnigg D. Ultrasound Markers in Fetal Hydronephrosis to Predict Postnatal Surgery. Ultraschall Med. 2018 Jul.

17. Lettgen B, Kropfl D, Bonzel KE, Meyer-Schwickerath M, Rascher W. Primary obstructed megaureter in neonates. Treatment by temporary uretero-cutaneostomy. Br J Urol. 1993 Nov;72(5 Pt 2):826-9.

18. Bakalis S, Peeva G, Gonzalez R, Poon LC, Nicolaides KH. Prediction of small-forgestational-age neonates: screening by biophysical and biochemical markers at 3034 weeks. Ultrasound Obstet Gynecol. 2015 Octl46(4):446-51

19. Lai J, Poon LCY, Pinas A, Bakalis S, Nicolaides KH. Uterine artery Doppler at 30-33 weeks' gestation in the prediction of preeclampsia. Fetal Diagn Ther. 2013;33(3):15663. 